\title{
Facile Hydrothermal Synthesis and Basic Gas-Sensing Properties of Two Three-Dimensional Nanostructures of $\mathrm{SnO}_{2}$
}

\author{
Lingna Xu, ${ }^{1}$ Weigen Chen, ${ }^{1,2}$ Caisheng Wang, ${ }^{2}$ Tuoyu Gao, ${ }^{1}$ and Qu Zhou ${ }^{1,3}$ \\ ${ }^{1}$ State Key Laboratory of Power Transmission Equipment \& System Security and New Technology, College of Electrical Engineering, \\ Chongqing University, Chongqing 400030, China \\ ${ }^{2}$ Department of Electrical and Computer Engineering, Wayne State University, Detroit, MI 48202, USA \\ ${ }^{3}$ College of Engineering and Technology, Southwest University, Chongqing 400715, China
}

Correspondence should be addressed to Lingna Xu; lingnaxu@cqu.edu.cn

Received 21 February 2014; Revised 8 May 2014; Accepted 15 May 2014; Published 2 June 2014

Academic Editor: Wen Zeng

Copyright (c) 2014 Lingna Xu et al. This is an open access article distributed under the Creative Commons Attribution License, which permits unrestricted use, distribution, and reproduction in any medium, provided the original work is properly cited.

\begin{abstract}
The hierarchical $\mathrm{SnO}_{2}$ sphere-like architecture, consisting of numerous thin nanosheets, was successfully synthesized via a facile hydrothermal method. The structures and morphologies of this hierarchical architecture were characterized in detail by means of powder X-ray diffraction (XRD), field-emission scanning electron microscopy (FE-SEM), and Brunauer-Emmett-Teller (BET). Further comparative experiments of gas-sensing performances of the as-prepared $\mathrm{SnO}_{2}$ were investigated towards ethanol. It shows this three-dimensional, sheet-spheres, $\mathrm{SnO}_{2}$ as a potential gas-sensing material for a broad range of future sensor applications, like sensitive response to other gases such as hydrogen, carbonic oxide, and methane.
\end{abstract}

\section{Introduction}

In the nanoscale, tremendous efforts have been paid to develop methods to adjust the microstructures of materials and thus tuning their functionalities [1-4]. Tin oxide $\left(\mathrm{SnO}_{2}\right)$, as an $n$-type semiconductor with a wide band gap $\left(E_{g}=\right.$ $3.6 \mathrm{eV}$, at $300 \mathrm{~K}$ ), has been the attractive material in various fields, such as gas sensors [5], catalysts [6], Li-ion batteries $[7,8]$, and electrode materials [9]. It has received considerable attention in both fundamental studies and practical applications due to unique properties such as photocatalysis and gas-sensing $[10,11]$. As we know, many fundamental physical and chemical properties of semiconductor materials rely not only on the composition but also on the structure, phase, shape, and size; synthesis of nanomaterials with a tunable shape or morphology has been the subject of intensive research [12-14]. Recently, numerous studies have demonstrated that materials with large surface area, efficient catalytic activity, and structural stability, such as threedimensional (3D) structures (e.g., spheres-like [15], aloeslike [16], and flowers-like [17]), exhibit enhanced gas-sensing performance. There are various preparation methods, such as thermal evaporation technique [18], sol-gel method [19], and hydrothermal route [20] which have been developed for 3D hierarchical architectures. Li et al. discuss porous $\mathrm{SnO}_{2}$ spheres which are synthesized through a solvothermal method [15]; Wang et al. use a sol-gel template synthetic technique to make $\mathrm{SnO}_{2}$ tubes [21]; Qi et al. study $\mathrm{SnO}_{2}$ fibers which are synthesized via a simple electrospinning method [22]. Among them, the hydrothermal method has been proved to be effective for preparation hierarchical $\mathrm{SnO}_{2}$ architectures [23]. Although the various nanostructure spheres of $\mathrm{SnO}_{2}$ have been synthesized, it still remains a huge challenge to shape-controlled routes with well-defined morphology and uniform size.

In this paper, we detailed a controllable synthesis of $\mathrm{SnO}_{2}$ sphere-like nanostructure through the straightforward hydrothermal process via the HMT (Hexamethylenetetramine) assisted. Both of these two $\mathrm{SnO}_{2}$ spheres samples exhibit the obvious high gas-sensing activities to ethanol during the experiments such as response and recovery time test, optimum operating temperature test, and different gas concentration test. Based on comparative studies, the possible formation mechanism was discussed basically. 
The gas-sensing properties of $\mathrm{SnO}_{2}$ sheet-spheres are found better than pointed columnar-spheres ones because of the higher voltage during the response-recovery time, lower working temperature, and more sensitive response in the same concentration to ethanol. The results not only provide a cheap and effective way to control the morphology and synthesize high performance three-dimensional sensing $\mathrm{SnO}_{2}$ to ethanol but also lay the foundation for the gassensitive reaction to the other gases (hydrogen, carbonic oxide, methane, etc.) for the future research.

\section{Experimental}

During the experiment, all chemicals are analytical-grade reagents from Chongqing Chuandong Chemical Reagent Co., Ltd., and used without any further purification.

Both of the $\mathrm{SnO}_{2}$ with nanostructures were compounded via the stable and controllable hydrothermal process.

2.1. Synthesis of $\mathrm{SnO}_{2}$ Sheet-Spheres. In a typical hydrothermal process, $0.53 \mathrm{~g} \mathrm{NaSnO}_{3} \cdot 3 \mathrm{H}_{2} \mathrm{O}(2 \mathrm{mmol})$ and $0.20 \mathrm{~g}$ $\mathrm{NaOH}(5 \mathrm{mmol})$ were dissolved into $75 \mathrm{~mL}$ deionized water. Then with magnetic continuously stirring, we got cleared solution. Next, 0.3 g HMT was dissolved into this cleared solution and stirred for next 30 minutes to get the reaction solution. Afterwards, the solution was transferred into a Teflon-lined stainless steel autoclave and maintained at $180^{\circ} \mathrm{C}$ for $24 \mathrm{~h}$. Finally, the product was collected by centrifugation, washed with deionized water and ethanol for six times, respectively, and finally dried at $60^{\circ} \mathrm{C}$ for $24 \mathrm{~h}$ to get the powder.

2.2. Synthesis of $\mathrm{SnO}_{2}$ Pointed Columnar-Spheres. Typically, $0.26 \mathrm{~g} \mathrm{SnCl}_{4} \cdot 5 \mathrm{H}_{2} \mathrm{O}, 0.32 \mathrm{~g} \mathrm{NaOH}$ and $0.10 \mathrm{~g} \mathrm{Na}_{2} \mathrm{SO}_{4}$ were dissolved into a $10 \mathrm{~mL}$ deionized water to stir for 5 minutes. Then $12 \mathrm{~mL}$ ethanol was dissolved into it to get white transparent suspension solution. After that, the evenly stirring mixtures were transferred into a Teflon-lined stainless steel autoclave and then heated in an oven at $180^{\circ} \mathrm{C}$ for $24 \mathrm{~h}$. After cooling down to room temperature naturally, the product was collected by centrifugation and washed six times with deionized water and absolute ethanol, subsequently. Finally, a white precipitate was harvested by glass culture dish and dried at $60^{\circ} \mathrm{C}$ overnight to get the solid powder.

2.3. Fabrication of Gas Sensor. The gas sensors were fabricated by dispersing the powders with ethanol to form pastes. The pastes were subsequently coated onto a ceramic tube to form a thin layer of sensing film (thickness about 10-20 $\mu \mathrm{m}$ ), positioned with a pair of Au electrodes at each end point. $\mathrm{A} \mathrm{Ni}-\mathrm{Cr}$ heating wire was inserted into the alumina tube to control the operating temperature by tuning the heating voltage. Figures 1(a) and 1(b) show a photograph of the sensor on the socket and a schematic image of the as-fabricated sensor, respectively. All the as-prepared sensors were finally aged at $240^{\circ} \mathrm{C}$ for $72 \mathrm{~h}$ to improve their stability and repeatability. The gas sensing properties of the samples were conducted using the Chemical Gas Sensor-8 (CGS-8) gas sensitivity instrument and analysis system (Beijing Elite Tech Co., Ltd.) that is a static test system with a heated area to place volatile liquid in the chamber. All the sensors were put into the test chamber and preheated at different operating temperature to keep steady. Then saturated target vapor was added which was the volatilization of the ethanol after heating. When the gas evenly filled the chamber, the system attained a new stable value. After that, the test chamber was opened to recover the sensor and repeated all the measurements several times to make sure the reproducibility of the gas-sensing response. The sensors are operated using a circuit voltage $\left(V_{c}\right)$, which was applied to allow for a measurement of the output voltage $\left(V_{\text {out }}\right)$ across. When air and ppm-level target gases flowed through the test chamber, the corresponding resistances of the sensor in air $\left(R_{a}\right)$ and in the presence of the target gas in air $\left(R_{t}\right)$ were measured by monitoring the $V_{\text {out }}$. Gassensing studies are carried out under laboratory condition with room temperature as $27^{\circ} \mathrm{C}$ and relative humidity as $40 \%$. Gas response in this paper is defined as $S=R_{a} / R_{t}$, in which $R_{a}$ and $R_{t}$ are the resistance of the sensor in air and in ethanol gas, respectively. And the response and recovery time are counted as the time taken by the sensor to achieve $90 \%$ of the total resistance change in the case of adsorption and desorption, respectively.

\section{Result and Discussion}

3.1. Structure Characterization. The phase purity of the prepared samples was characterized by X-ray diffraction (XRD, scanning rate: $0.02^{\circ} \mathrm{s}^{-1}$ in the 2 -theta range of $\left.10-90^{\circ}\right)$. All the diffraction peaks of the sample in Figure 2 were well indexed to the $\mathrm{SnO}_{2}$ with the rutile structure (space group: $\mathrm{P} 42$, JCPDS card number 41-1445) and no obvious characteristic peaks from other impurities were detected, indicating the high purity and crystallinity of the final products. It is easy to see that both two samples have similar XRD patterns such as location, intensity, and breadth, suggesting that the samples exhibit almost the same crystal size. Meanwhile, the structure of the solid powder was investigated using a Nova 400 Nano field emission scanning electronic microscopy (FE-SEM).

Figure 3(a) displays the low-magnification SEM images of the sheet-spheres samples. It shows that the $\mathrm{SnO}_{2}$ sheetspheres products exhibit a unique hierarchical sheet architecture, and these spheres dispersed in the samples with a diameter about $700 \mathrm{~nm}$. An enlarged SEM image of an individual sphere-like architecture is shown in Figure 3(b), which apparently shows that the unique hierarchical spherelike architecture is regularly composed of numerous wellordered thin nanosheets. Figures 3(c) and 3(d) show the $\mathrm{SnO}_{2}$ pointed columnar-spheres in $1 \mu \mathrm{m}$ and $500 \mathrm{~nm}$, respectively, and the diameter of it is larger than the sheet-sphere-like one.

Based on the experimental observations and analysis, we propose a plausible growth mechanism for the morphologies evolution of the $\mathrm{SnO}_{2}$, as shown in Figure 4. The $\mathrm{SnO}_{2}$ nanocrystal was formed from the hydrolysis of $\mathrm{Sn}^{2+}$ ions. To eliminate the surface energy, the $\mathrm{SnO}_{2}$ colloids were next aggregated to form nanosheets and finally formed hierarchical sphere-like architectures. In our study, the HMT plays a key role in the formation and the space distribution of 


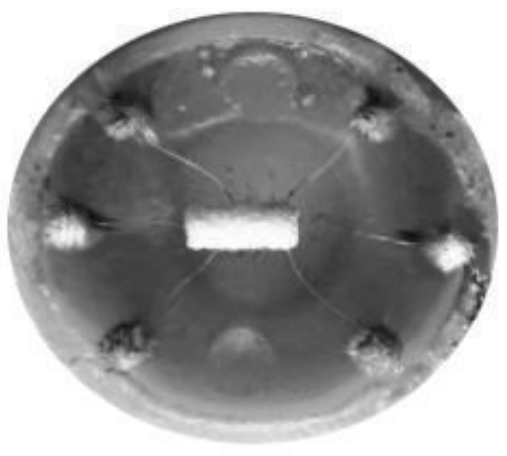

(a)

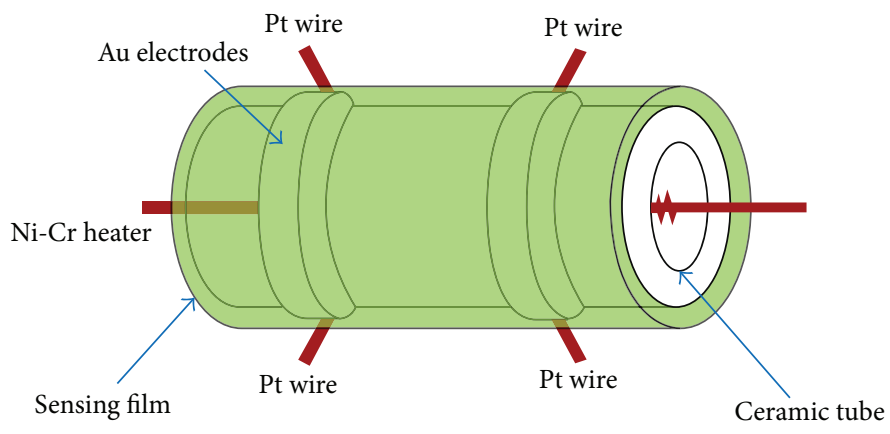

(b)

FIgURE 1: (a) Fabricated sensor and (b) schematic diagram of indirect-heating sensor.

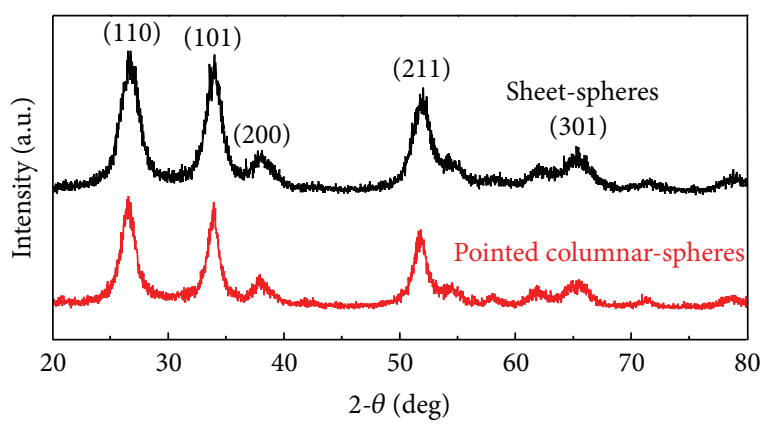

FIGURE 2: XRD patterns of the samples.

TABLE 1: BET surface area and pore structure parameters.

\begin{tabular}{lccc}
\hline Sample & $S_{\mathrm{BET}}\left(\mathrm{m}^{2} / \mathrm{g}\right)$ & $V_{p}\left(\mathrm{~cm}^{3} / \mathrm{g}\right)$ & $d_{p}(\mathrm{~nm})$ \\
\hline Sheet-spheres & 22.3 & 0.04 & 23.8 \\
Pointed columnar-spheres & 17.8 & 0.02 & 23.9 \\
\hline
\end{tabular}

the precursor. A large number of $\mathrm{Sn}^{2+}$ ions accumulated on the long chains of HMT in the solution and decreased the concentration of free $\mathrm{Sn}^{2+}$ ion; thus, HMT provided many nucleation sites; with reaction time passing by, numerous tiny $\mathrm{SnO}_{2}$ crystals nucleated on HMT chains and grew further, which resulted in the formation of $\mathrm{SnO}_{2}$ nanosheets.

The main factors that affect the sensing mechanism qualitatively are the specific surface area and pore structure of the materials, which can be measured using the BET method [24]. As shown in Table 1, the obtained specific surface areas for the sheet-spheres and pointed columnar-spheres are $22.3 \mathrm{~m}^{2} / \mathrm{g}$ and $17.8 \mathrm{~m}^{2} / \mathrm{g}$. What counts is that the larger specific surface area may facilitate the interaction between the material surface and the gas molecule, which triggers even more substantial output in electric signal. This, together with the scale of surface pores, suggests that the sheet-spheres of $\mathrm{SnO}_{2}$ may exhibit a better sensing behavior.
3.2. Gas Sensing Mechanism. It is well known that the $\mathrm{SnO}_{2}$ belongs to an $n$-type semiconductor and its sensing character is governed by the change of surface resistance; the species and amount of chemisorbed oxygen on surface are critical for the variation in resistance. As the sensor was first aged in air, oxygen can be absorbed on its surface and can act as a trap for the conduction band electrons of $\mathrm{SnO}_{2}$ due to their strong electronegativity, which would be ionized to $\mathrm{O}^{2-}, \mathrm{O}^{-}$, or $\mathrm{O}_{2}{ }^{-}$by capturing free electrons from the conduction band of $\mathrm{SnO}_{2}$. The entire adsorption and reaction process can be expressed as follows [25-29]:

$$
\begin{gathered}
\mathrm{O}_{2} \text { (gas) } \longrightarrow \mathrm{O}_{2} \text { (ads) } \\
\mathrm{O}_{2}(\text { ads })+\mathrm{e}^{-} \longrightarrow \mathrm{O}_{2}^{-} \text {(ads) } \\
\mathrm{O}_{2}^{-} \text {(ads) }+\mathrm{e}^{-} \longrightarrow 2 \mathrm{O}^{-} \text {(ads) } \\
\mathrm{O}^{-} \text {(ads) }+\mathrm{e}^{-} \longrightarrow \mathrm{O}^{2-} \text { (ads) }
\end{gathered}
$$

The absorbed oxygen could cause a depletion layer and band bending on the surface, which consequently increases the energy barrier, namely, the resistance of the sensing material $\mathrm{SnO}_{2}$. As the $\mathrm{C}_{2} \mathrm{H}_{5} \mathrm{OH}$ is introduced, chemical reactions take place between the $\mathrm{C}_{2} \mathrm{H}_{5} \mathrm{OH}$ and the ionized oxygen $\mathrm{O}_{2}$, which gives out electrons back to the $\mathrm{SnO}_{2}$ surface. The possible reactions are summarized by the following equations:

$$
\begin{gathered}
\mathrm{C}_{2} \mathrm{H}_{5} \mathrm{OH} \text { (gas) }+\mathrm{O}^{2-} \text { (ads) } \\
\longrightarrow \mathrm{C}_{2} \mathrm{H}_{5} \mathrm{O}^{-} \text {(gas) }+\mathrm{OH}^{-} \text {(ads) } \\
2 \mathrm{C}_{2} \mathrm{H}_{5} \mathrm{O}^{-} \text {(gas) } \longrightarrow\left(\mathrm{C}_{2} \mathrm{H}_{5}\right)_{2} \mathrm{O} \text { (ads) }+\mathrm{O}^{-} \text {(ads) }+\mathrm{e}^{-}, \\
\mathrm{C}_{2} \mathrm{H}_{5} \mathrm{OH} \text { (gas) }+\mathrm{O}^{2-}(\text { ads }) \longrightarrow \mathrm{C}_{2} \mathrm{H}_{4} \mathrm{O}^{2-} \text { (ads) }+\mathrm{H}_{2} \mathrm{O}, \\
\mathrm{C}_{2} \mathrm{H}_{4} \mathrm{O}^{-} \text {(ads) } \longrightarrow \mathrm{CH}_{3} \mathrm{CHO} \text { (ads) }+\mathrm{e}^{-}, \\
\mathrm{CH}_{3} \mathrm{CHO} \text { (ads) }+5 \mathrm{O}^{2-} \text { (ads) } \longrightarrow 2 \mathrm{CO}_{2}+2 \mathrm{H}_{2} \mathrm{O}+10 \mathrm{e}^{-} .
\end{gathered}
$$




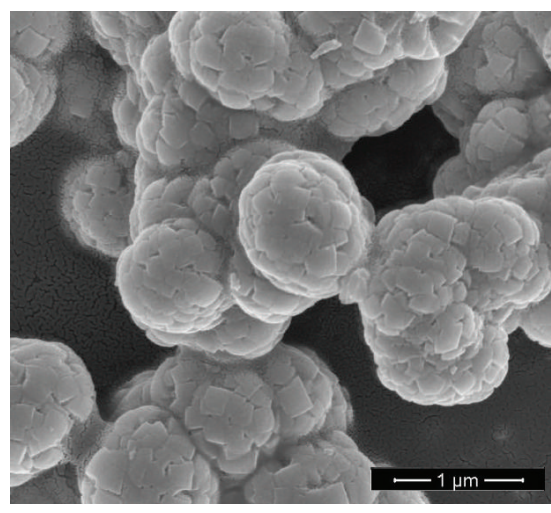

(a)

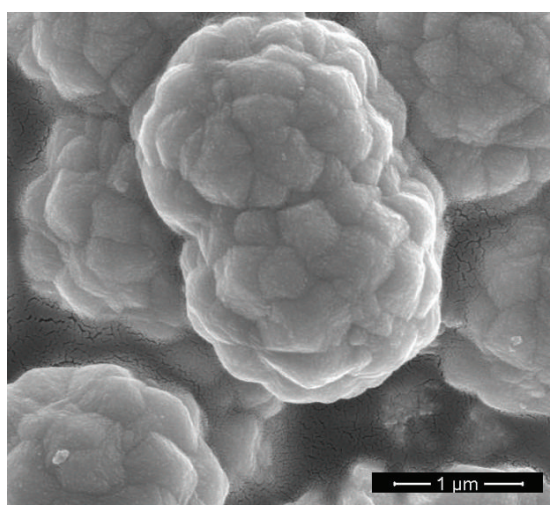

(c)

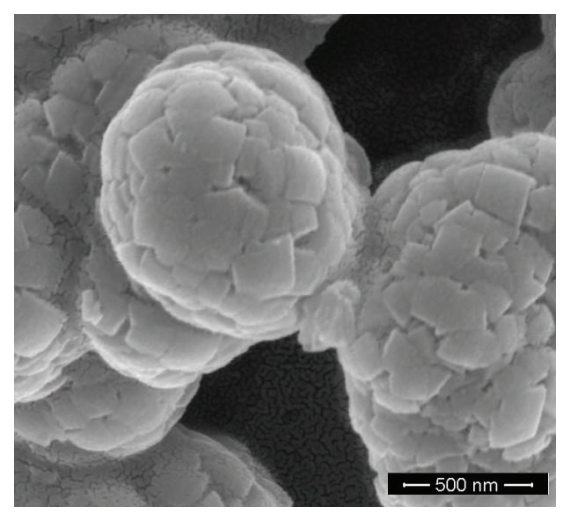

(b)

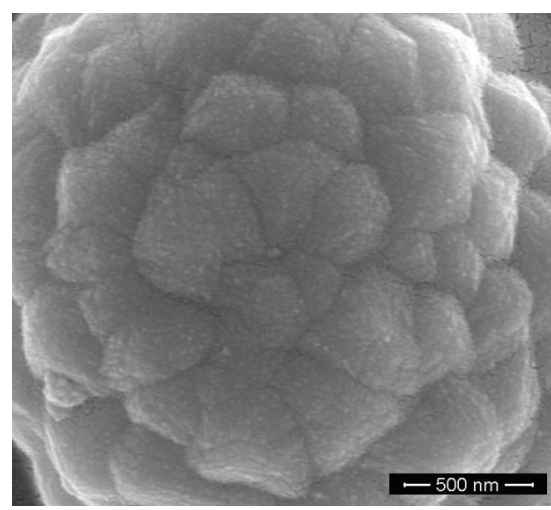

(d)

FIGURE 3: SEM images of the sheet-spheres and pointed columnar-spheres: (a) $1 \mu \mathrm{m}$, (b) $500 \mathrm{~nm}$, (c) $1 \mu \mathrm{m}$, and (d) $500 \mathrm{~nm}$.

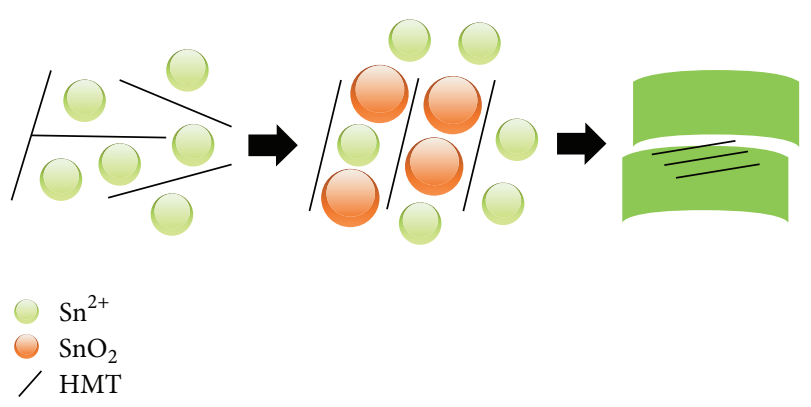

FIGURE 4: Schematic illustration of the evolution processes of the $\mathrm{SnO}_{2}$ sheet-spheres.

In general, there are two types of reactions occurring simultaneously on the oxide surface: (1) the target gas molecules react with the preabsorbed oxygen, and (2) the target gas molecules react directly with the surface atoms of oxide. Whatever the reaction type, the electrons that are produced from these reactions would decrease the resistance significantly, which results in drastic increase of output voltage, as generally observed at the working stage of a sensor in Figure 5. It presents a representative response-recovery characteristic for the sensor operated at $340^{\circ} \mathrm{C}$ under ethanol gas concentration of $100 \mathrm{ppm}$. The response and recovery times are two key quantities for a sensor, which are defined as the time needed

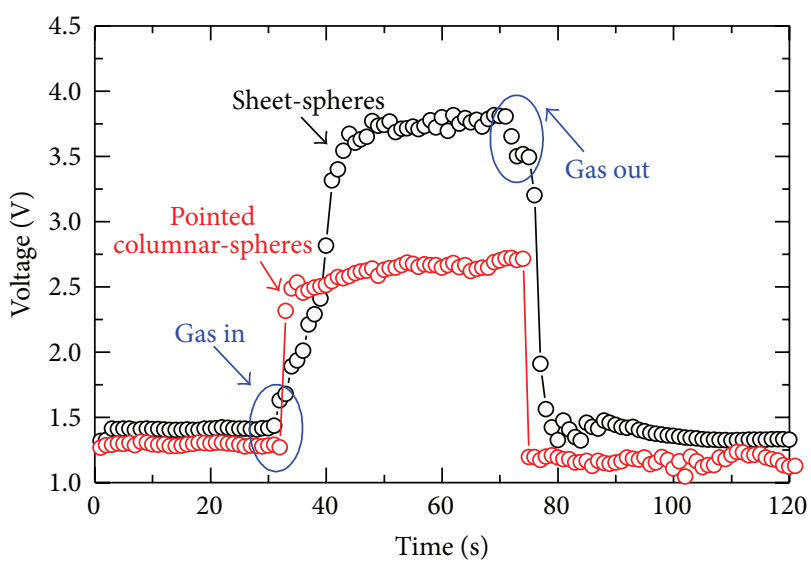

FIgURE 5: Response-recovery characteristics of the sensors at $340^{\circ} \mathrm{C}$ under 100 ppm ethanol.

to reach $90 \%$ response or recovery when gas is in or out. Under the definition described above, the response and recovery times for the sheet-spheres and pointed columnarspheres are evaluated to be about 21-23 s and 19-21 s, which meet the basic demands for an industrial application. As seen in Figure 5, voltages of both two kinds of samples increase when gas is in but return to their original state when gas is out. The major difference among the sensors is that the voltage 


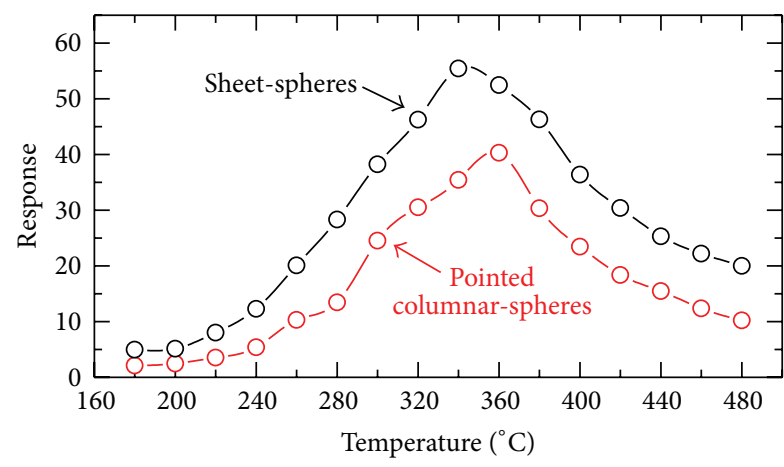

Figure 6: Gas response of the sensors to different operating temperature under $250 \mathrm{ppm}$ ethanol.

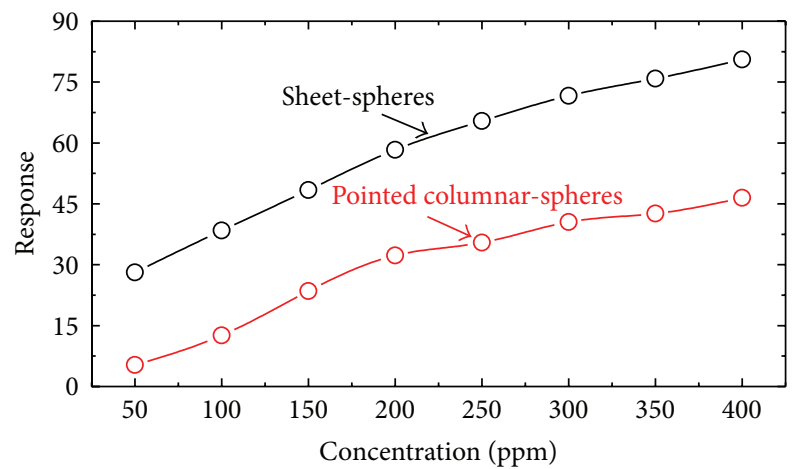

FIGURE 7: Gas response of the sensors to different gas concentration ranking from 50 to $400 \mathrm{ppm}$.

for the sheet-spheres is substantially larger than that for the pointed columnar-spheres sensor at working stage, verifying its better sensing performances.

To investigate how the various morphologies of $\mathrm{SnO}_{2}$ impact their gas sensing properties, we further make a systematic comparison of the gas sensing performance of these two 3-dimensional $\mathrm{SnO}_{2}$ nanostructure materials.

Obviously, the response of the sensors increases with a raise of temperature till reaching the maximum value and then decreases with any further increase of temperature. The gas response to $250 \mathrm{ppm}$ ethanol was firstly investigated at different operating temperatures and the result is shown in Figure 6. One can see that sheet-spheres $\mathrm{SnO}_{2}$ exhibit exclusive better sensing performances to ethanol than that of pointed columnar-spheres. The highest gas response to the ethanol is estimated to be 55 for $\mathrm{SnO}_{2}$ sheet-spheres, obviously higher than 40 for pointed columnar-nanospheres case. Moreover, we further determine the optimum working temperature of sheet-spheres sensor to be about $340^{\circ} \mathrm{C}$, lower than that of the pointed columnar-spheres sensor with value of $360^{\circ} \mathrm{C}$.

To further investigate how the gas concentration affects the gas response, we present in Figure 7 the gas response to ethanol with operating temperature of $340^{\circ} \mathrm{C}$. Independent of the sensor types, the gas response increases dramatically with no sign of saturation, as the concentration ranges from
50 to $400 \mathrm{ppm}$. We find again that in the two of the samples, the sheet-spheres show higher response in the measured concentrations, although it is well known that the response may be affected by the concentration of examined gas. Further, we notice that, independent of the samples, the response increases in a nonlinear manner with the rise of gas concentration, which can be explained from the gas-diffusion theory [30].

According to the measurement above, we thus conclude that the 3-dimensional $\mathrm{SnO}_{2}$ for sheet-spheres exhibit distinctive better sensing properties than that of the pointed columnar-spheres.

\section{Conclusions}

In summary, we have prepared nanostructure $\mathrm{SnO}_{2}$ sheetspheres and pointed columnar-spheres through the effective hydrothermal method and investigated their structures and gas sensing properties. In particular, we compared systematically the ethanol sensing properties between these two $3 \mathrm{D} \mathrm{SnO}_{2}$. The results show that $\mathrm{SnO}_{2}$ sheet-spheres exhibit higher gas response to ethanol as well as lower working temperature as compared to that of pointed columnar-spheres. These results indicate that the gas-sensing properties of $\mathrm{SnO}_{2}$ materials can be enhanced by controlling their shapes and surface structures. Also these samples lay the foundation for the gas-sensitive reaction to other gases (hydrogen, carbonic oxide, methane, etc.) for the future research.

\section{Conflict of Interests}

The authors declare that there is no conflict of interests regarding the publication of this paper.

\section{Acknowledgments}

The authors appreciate the financial supports of the National Natural Science Fund of China (nos. 51277185 and 51202302) and the National Special Fund for Major Research Instrumentation Development (no. 2012YQ16000705).

\section{References}

[1] C. B. Murray, D. J. Norris, and M. G. Bawendi, "Synthesis and characterization of nearly monodisperse CdE (E = sulfur, selenium, tellurium) semiconductor nanocrystallites," Journal of the American Chemical Society, vol. 115, no. 19, pp. 8706-8715, 1993.

[2] X. Wang, J. Zhuang, Q. Peng, and Y. Li, "A general strategy for nanocrystal synthesis," Nature, vol. 437, no. 7055, pp. 121-124, 2005.

[3] J. Hu, L.-S. Li, W. Yang, L. Manna, L.-W. Wang, and A. P. Alivisatos, "Linearly polarized emission from colloidal semiconductor quantum rods," Science, vol. 292, no. 5524, pp. 20602063, 2001.

[4] A. P. Alivisatos, "Semiconductor clusters, nanocrystals, and quantum dots," Science, vol. 271, no. 5251, pp. 933-937, 1996. 
[5] W. Zeng, B. Miao, Q. Zhou, and L. Lin, "Hydrothermal synthesis and gas sensing properties of variety low dimensional nanostructures of $\mathrm{SnO}_{2}$," Physica E, vol. 47, pp. 116-121, 2013.

[6] K.-I. Shimizu, M. Katagiri, S. Satokawa, and A. Satsuma, "Sintering-resistant and self-regenerative properties of $\mathrm{Ag} / \mathrm{SnO}_{2}$ catalyst for soot oxidation," Applied Catalysis B: Environmental, vol. 108-109, pp. 39-46, 2011.

[7] Y. Idota, T. Kubota, A. Matsufuji, Y. Maekawa, and T. Miyasaka, "Tin-based amorphous oxide: a high-capacity lithium-ionstorage material," Science, vol. 276, no. 5317, pp. 1395-1397, 1997.

[8] M.-S. Park, G.-X. Wang, Y.-M. Kang, D. Wexler, S.-X. Dou, and H.-K. Liu, "Preparation and electrochemical properties of $\mathrm{SnO}_{2}$ nanowires for application in lithium-ion batteries," Angewandte Chemie-International Edition, vol. 46, no. 5, pp. 750-753, 2007.

[9] Z. Wen, F. Zheng, and K. Liu, "Synthesis of porous $\mathrm{SnO}_{2}$ nanospheres and their application for lithium-ion battery," Materials Letters, vol. 68, pp. 469-471, 2012.

[10] N. D. Khoang, D. D. Trung, N. van Duy, N. D. Hoa, and N. van Hieu, "Design of $\mathrm{SnO}_{2} / \mathrm{ZnO}$ hierarchical nanostructures for enhanced ethanol gas-sensing performance," Sensors and Actuators B: Chemical, vol. 174, pp. 594-601, 2012.

[11] H. Li, F. Meng, J. Liu et al., "Synthesis and gas sensing properties of hierarchical meso-macroporous $\mathrm{SnO}_{2}$ for detection of indoor air pollutants," Sensors and Actuators B: Chemical, vol. 166-167, pp. 519-525, 2012.

[12] J. Y. Park, S.-W. Choi, and S. S. Kim, "Junction-tuned $\mathrm{SnO}_{2}$ nanowires and their sensing properties," Journal of Physical Chemistry C, vol. 115, no. 26, pp. 12774-12781, 2011.

[13] Q. Zhou, W. Chen, L. Xu, and S. Peng, "Hydrothermal synthesis of various hierarchical $\mathrm{ZnO}$ nanostructures and their methane sensing properties," Sensors, vol. 13, no. 5, pp. 6171-6182, 2013.

[14] L. Shi and H. Lin, "Preparation of band gap tunable $\mathrm{SnO}_{2}$ nanotubes and their ethanol sensing properties," Langmuir, vol. 27, no. 7, pp. 3977-3981, 2011.

[15] Z. Li, Q. Zhao, W. Fan, and J. Zhan, "Porous $\mathrm{SnO}_{2}$ nanospheres as sensitive gas sensors for volatile organic compounds detection," Nanoscale, vol. 3, no. 4, pp. 1646-1652, 2011.

[16] L. Mei, J. Deng, X. Yin et al., "Ultrasensitive ethanol sensor based on 3D aloe-like $\mathrm{SnO}_{2}$," Sensors and Actuators B: Chemical, vol. 166-167, pp. 7-11, 2012.

[17] H. Wang, Q. Liang, W. Wang, Y. An, J. Li, and L. Guo, "Preparation of flower-like $\mathrm{SnO}_{2}$ nanostructures and their applications in gas-sensing and lithium storage," Crystal Growth and Design, vol. 11, no. 7, pp. 2942-2947, 2011.

[18] Z. R. Dai, Z. W. Pan, and Z. L. Wang, "Growth and structure evolution of novel tin oxide diskettes," Journal of the American Chemical Society, vol. 124, no. 29, pp. 8673-8680, 2002.

[19] Q. Dong, H. Su, J. Xu, and D. Zhang, "Influence of hierarchical nanostructures on the gas sensing properties of $\mathrm{SnO}_{2}$ biomorphic films," Sensors and Actuators B: Chemical, vol. 123, no. 1, pp. 420-428, 2007.

[20] M. Wu, W. Zeng, and Y. Li, "Hydrothermal synthesis of novel $\mathrm{SnO}_{2}$ nanoflowers and their gas-sensing properties," Materials Letters, vol. 104, pp. 34-36, 2013.

[21] G. X. Wang, J. S. Park, M. S. Park, and X. L. Gou, "Synthesis and high gas sensitivity of tin oxide nanotubes," Sensors and Actuators B: Chemical, vol. 131, no. 1, pp. 313-317, 2008.

[22] Q. Qi, T. Zhang, L. Liu, X. Zheng, and G. Lu, "Improved $\mathrm{NH}_{3}, \mathrm{C}_{2} \mathrm{H}_{5} \mathrm{OH}$, and $\mathrm{CH}_{3} \mathrm{COCH}_{3}$ sensing properties of $\mathrm{SnO}_{2}$ nanofibers by adding block copolymer P123," Sensors and Actuators B: Chemical, vol. 141, no. 1, pp. 174-178, 2009.
[23] H. Zhang, Q. He, X. Zhu, D. Pan, X. Deng, and Z. Jiao, "Surfactant-free solution phase synthesis of monodispersed $\mathrm{SnO}_{2}$ hierarchical nanostructures and gas sensing properties," CrystEngComm, vol. 14, no. 9, pp. 3169-3176, 2012.

[24] W. Zeng, T. Liu, and Z. Wang, "Impact of Nb doping on gassensing performance of $\mathrm{TiO}_{2}$ thick-film sensors," Sensors and Actuators B: Chemical, vol. 166-167, pp. 141-149, 2012.

[25] W. G. Chen, Q. Zhou, and L. N. Xu, "Improved methane sensing properties of Co-doped $\mathrm{SnO}_{2}$ electrospun nanofibers," Journal of Nanomaterials, vol. 2013, Article ID 173232, 9 pages, 2013.

[26] Z. Weng and L. Tian-mo, "Gas-sensing properties of $\mathrm{SnO}_{2}$ $\mathrm{TiO}_{2}$-based sensor for volatile organic compound gas and its sensing mechanism," Physica B: Condensed Matter, vol. 405, no. 5, pp. 1345-1348, 2010.

[27] N. Bârsan, M. Hübner, and U. Weimar, "Conduction mechanisms in $\mathrm{SnO}_{2}$ based polycrystalline thick film gas sensors exposed to $\mathrm{CO}$ and $\mathrm{H}_{2}$ in different oxygen backgrounds," Sensors and Actuators B: Chemical, vol. 157, no. 2, pp. 510-517, 2011.

[28] M. Hübner, R. G. Pavelko, and N. Barsan, "Influence of oxygen backgrounds on hydrogen sensing with $\mathrm{SnO}_{2}$ nanomaterials," Sensors and Actuators B: Chemical, vol. 154, no. 2, pp. 264-269, 2011.

[29] W. G. Chen, Q. Zhou, and S. D. Peng, "Hydrothermal synthesis of Pt-, Fe-, and $\mathrm{Zn}$-doped $\mathrm{SnO}_{2}$ nanospheres and carbon monoxide sensing properties," Advances in Materials Science and Engineering, vol. 2013, Article ID 578460, 8 pages, 2013.

[30] G. Sakai, N. Matsunaga, K. Shimanoe, and N. Yamazoe, “Theory of gas-diffusion controlled sensitivity for thin film semiconductor gas sensor," Sensors and Actuators B: Chemical, vol. 80, no. 2, pp. 125-131, 2001. 

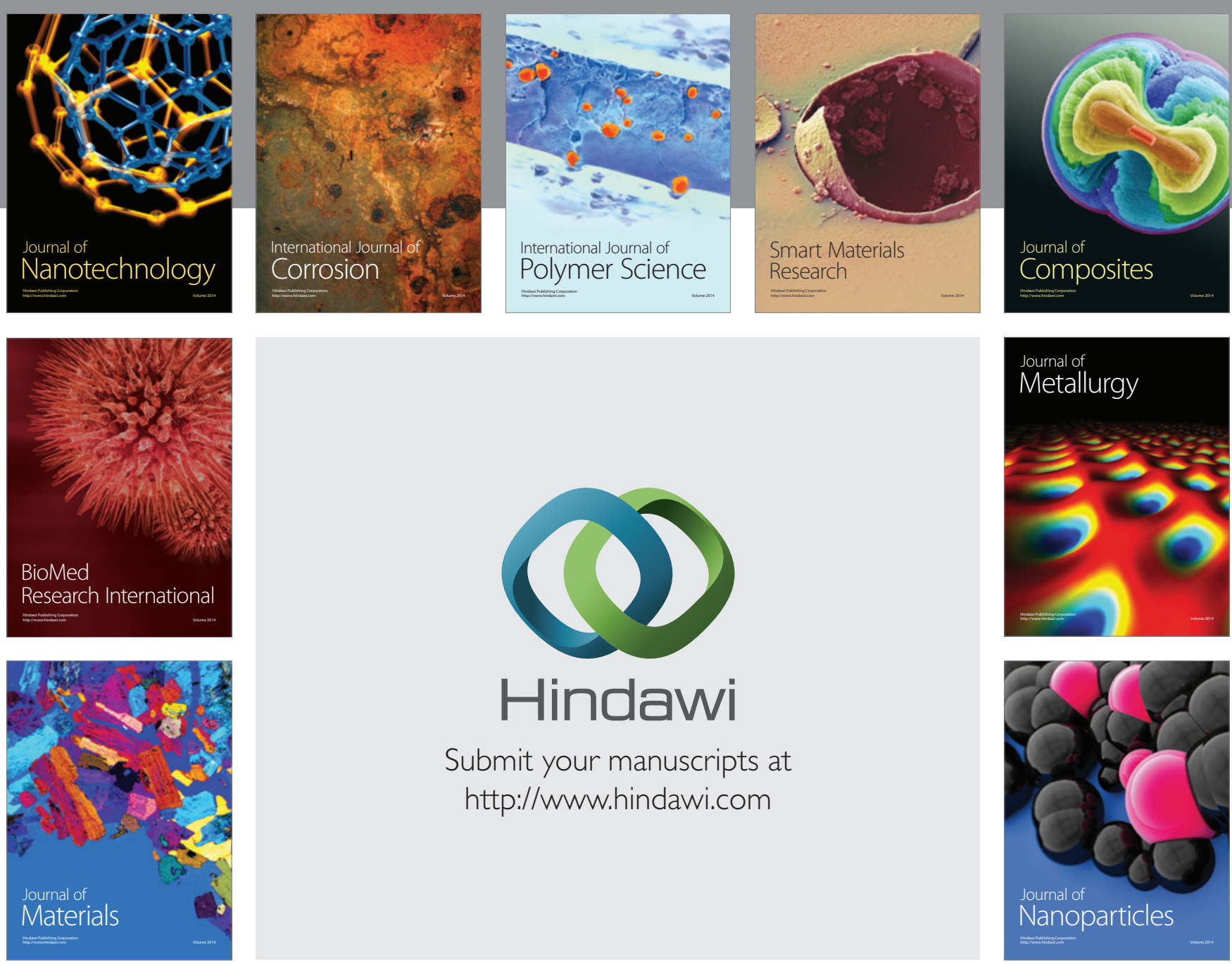

Submit your manuscripts at http://www.hindawi.com
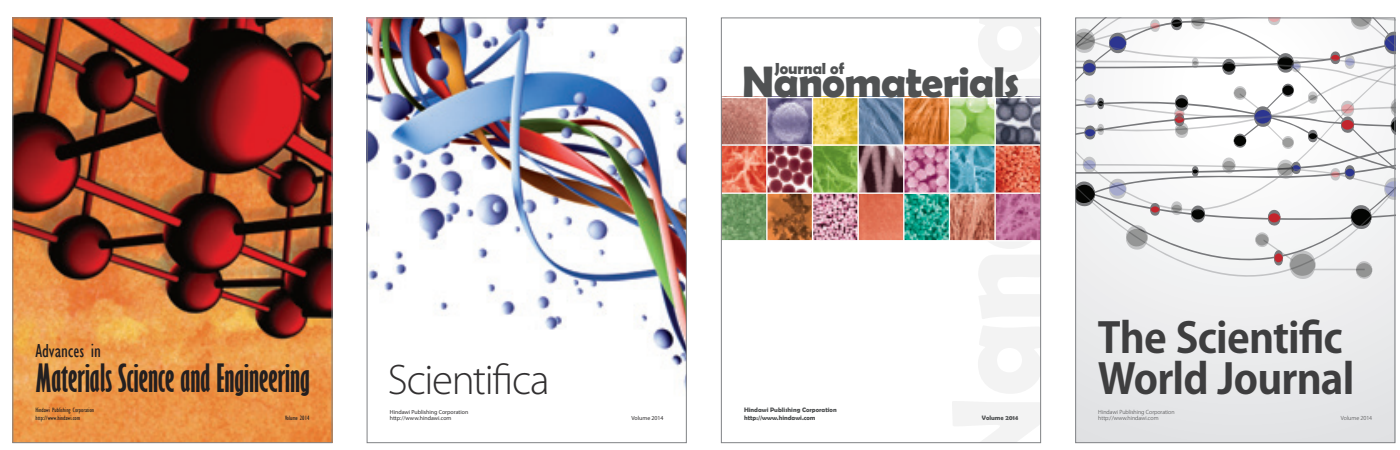

\section{The Scientific World Journal}
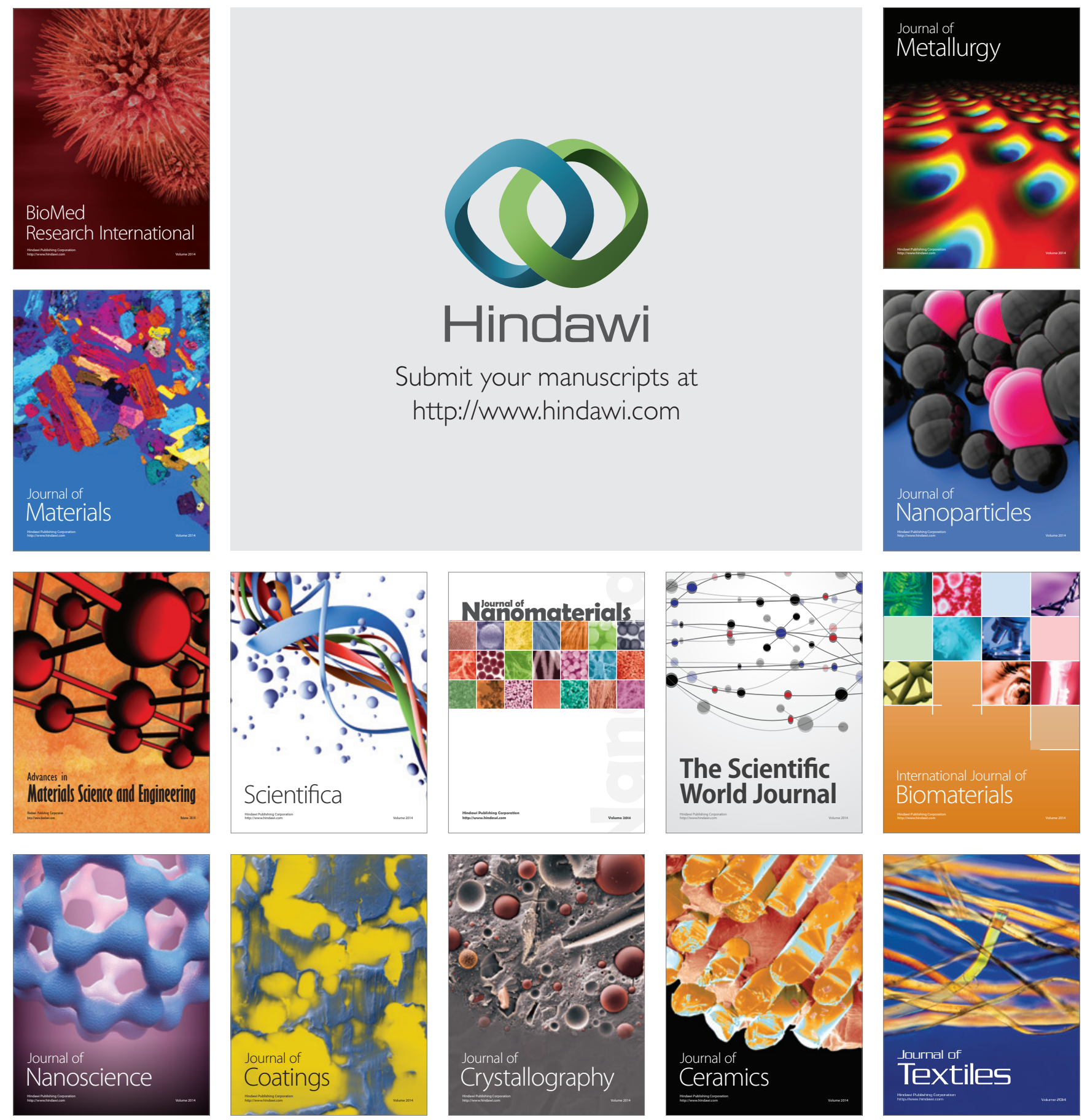